\title{
A Novel Application of an Adjustable Catheter in Acute Radicular Pain Management
}

Maria Clemente $\cdot$ Carla Roero $\cdot$ Veronica Perlo • Elena Peila •

Anna De Luca

Received: November 11, 2018 / Published online: January 8, 2019

(C) The Author(s) 2019

\section{ABSTRACT}

Introduction: Acute lumbosacral radicular syndrome is often a medical disorder of difficult management. Epidural steroid injection is a useful approach for the herniated disc and radiculitis. The transforaminal approach is usually considered more effective and targetspecific, but it can be associated with permanent lower extremity paralysis. A caudal approach with an adjustable catheter has been widely used in adhesiolysis in chronic low back pain, but there are no reports of its application in acute radicular pain. The aim of this study is to assess the clinical effectiveness of epidural steroid injection by caudal approach with an adjustable catheter in patients with severe acute radicular pain.

Methods: Fifty-five patients with severe acute radiculopathy were treated with epidural steroid injection by an epidural catheter whose tip can be directed laterally on the selected site. Numerical rating scale (NRS), pain relief, and analgesic consumption were observed after 1, 3,

Enhanced Digital Features To view enhanced digital features for this article go to: https://doi.org/10.6084/ m9.figshare.7484882.

M. Clemente $(\bowtie) \cdot$ C. Roero · V. Perlo · E. Peila . A. De Luca

Pain Clinic and Palliative Care Unit, A.O.U. Città della Salute e della Scienza, Corso Bramante 88, 10131 Turin, Italy

e-mail: mcclemente@libero.it
6, and 12 months. Analgesic consumption (AC) and functional recovery (FR) have been considered secondary outcomes.

Results: We observed a significant reduction of NRS score that was constant every 12 months. Pain relief was good after 1 month and improved further after 3 months. Only a few patients perceived poor pain relief and only three patients relapsed. More than $70 \%$ of the patients were drug-free at the 12th month.

Conclusions: The caudal approach with adjustable catheter showed similar but more lasting effects on the acute severe radicular pain when compared to other epidural injections techniques; it is extremely target-specific and thus allows the use of small doses of corticosteroids; moreover, the adjustable catheter makes the procedure free from the risk of major complications.

Funding: No funding or sponsorship was received for this study. Sponsorship for article publication fees were funded by TSS Medical SRL.

Keywords: Acute pain; Acute radiculopathy; Catheter; Epidural; Target-specific

\section{INTRODUCTION}

Acute lumbosacral radicular syndrome is a medical disorder of often difficult management, which usually involves young patients [1]. 
Pharmacological treatment is mostly ineffective or unsatisfactory [2]. Pain typically involves an area served by a nerve root or sacral spine [1]. The most common cause of this syndrome is a herniated disc.

Epidural steroid injection is a useful approach to the herniated disc and radiculitis, and it can be performed by caudal, interlaminar, or transforaminal approaches [3].

The recommendation of the evidence-based guidelines for the diagnosis and interventional treatment of spinal pain is one of the three that may be effectively used [4]. However, the transforaminal one is usually considered more effective and target-specific because it allows reaching the targeted pain generators in comparison to intralaminar or sacral injections. Unfortunately, this technique is linked to a major complication, which is permanent lowerextremity paralysis as the consequence of compression or transection of the artery of Adamkiewicz or the radicular artery [5-7]. This complication has been described in tomography-guided procedures, too [7]. The caudal epidural approach is the safest because the catheter is inserted in sacral hiatus and does not interfere with radicular artery in the foramen. This approach provides a good analgesic effect, however it is not so target-specific as the transforaminal approach [6].

Many studies display different epidural injection techniques but no data has been published on epidural selective injection through adjustable catheter.

Some authors report the application of adjustable catheter only in percutaneous adhesiolysis for managing chronic back pain in patients affected by lumbar spinal canal stenosis $[8,9]$, but not in acute radicular pain.

The aim of this study is to find a safe, effective, and target-specific technique for epidural steroid injection to manage acute severe radicular pain.

\section{METHODS}

The study was not registered at ClinicalTrials.gov identifier because it is a retrospective study, and not a randomized controlled trial (RCT).
All procedures performed in the study were in accordance with the 1964 Helsinki Declaration and its later amendments or comparable ethical standards. Patients gave their informed consent for participation in the research study.

\section{Participants}

Fifty-five subjects with severe radicular pain [male/female: 28 (50.9\%)/27 (49.1\%); age $58.4+13.9$ ] were recruited between February 2013 and February 2015, at the Pain Therapy and Palliative Care center (Table 1).

Subjects reported acute, moderate-to-severe and drug-resistant radicular pain (numeric rating scale, NRS: $>5$ ) with consequently daily function impairment [10] at one or two consecutive root fields and lumbosacral magnetic resonance, electromyography, and clinical findings showed herniated lumbar disc.

The pain symptom did not improve after at least 1 week of conventional medical treatment: steroids (dexamethasone $4-8 \mathrm{mg}$ intramuscular injection), non-steroidal anti-inflammatory and opioids) $[4,11]$. Patients affected by infectious or hemorrhagic diseases, spinal trauma or tumor, and patients assuming anticoagulant or antiaggregant therapy that could not be replaced by heparin were excluded from the study.

\section{Intervention}

All patients were evaluated through a complete medical examination performed by an expert physician. The following tests were performed: complete interview for medical history and associated diseases (diabetes, coagulopathies, infections, tumor diseases), heart and lung examination, neurological examination, and pain assessment.

A blood sample was obtained for platelet counts and other parameters of coagulation. Chronic anticoagulant or antiaggregant therapies were replaced by low molecular weight heparin.

All patients have been treated in a day surgery regimen. Relaxation was induced using hypnosis techniques. Relaxation was induced with progressive muscle relaxation therapy and 
Table 1 Patients' characteristics

\begin{tabular}{llll}
\hline & Mean $(\mathbf{S D}) / N(\%)$ & Mean $($ SD) $/ \boldsymbol{N}(\%)$ \\
\hline Age (years) & $58.4(13.9)$ & Basal NRS & $7.5(1.9)$ \\
Gender & & Side: & $23(46)$ \\
M (\%) & $28(50.9)$ & Right $(\%)$ & $27(54)$ \\
F (\%) & $27(49.1)$ & Left $(\%)$ & $5(9.1)$ \\
L3 & $5(9.6)$ & Complications & $3(5.5)$ \\
L4 & $14(26.6)$ & & Suspended procedures \\
L5 & $30(57.7)$ & & \\
S1 & $3(5.8)$ & & \\
\hline
\end{tabular}

$N R S$ numerical rating scale, $D S$ standard deviation

autogenous training. After intravenous access was inserted, the patient was accepted in the operating room. Here, the patient was placed in prone position. Cardiac activity, oxygen saturation, and blood pressure were continuously monitored.

The procedure was performed with the St. Reed kit (Seawon Meditech CO., limited, Siheung-Si, Gyeonggi-do, Korea), which contains a 15-gauge bone marrow needle $103 \mathrm{~mm}$ long, covered by a plastic cannula and an epidural catheter whose tip can be directed laterally (external diameter $1.6 \mathrm{~mm}$; length $300 \mathrm{~mm}$ ). The catheter is connected to a holder with a catheter controller allowing to move and maintain the tip of the catheter on the desired side (Fig. 1a, b).

After local anesthesia with lidocaine $2 \%$, the needle was introduced in the hiatus sacralis. Its position was checked by fluoroscopic control in antero-posterior and latero-lateral vision.

The needle was removed leaving the plastic cannula and the catheter was introduced trough the sacral epidural space. Under fluoroscopic control, the catheter was advanced until the radicular target (Fig. 1). The guidewire in the catheter was removed and $1 \mathrm{ml}$ of saline solution with $25 \mathrm{mg}$ of hydrocortisone was injected, followed by another $2 \mathrm{ml}$ of saline solution to allow the drug to completely exit the catheter tip.
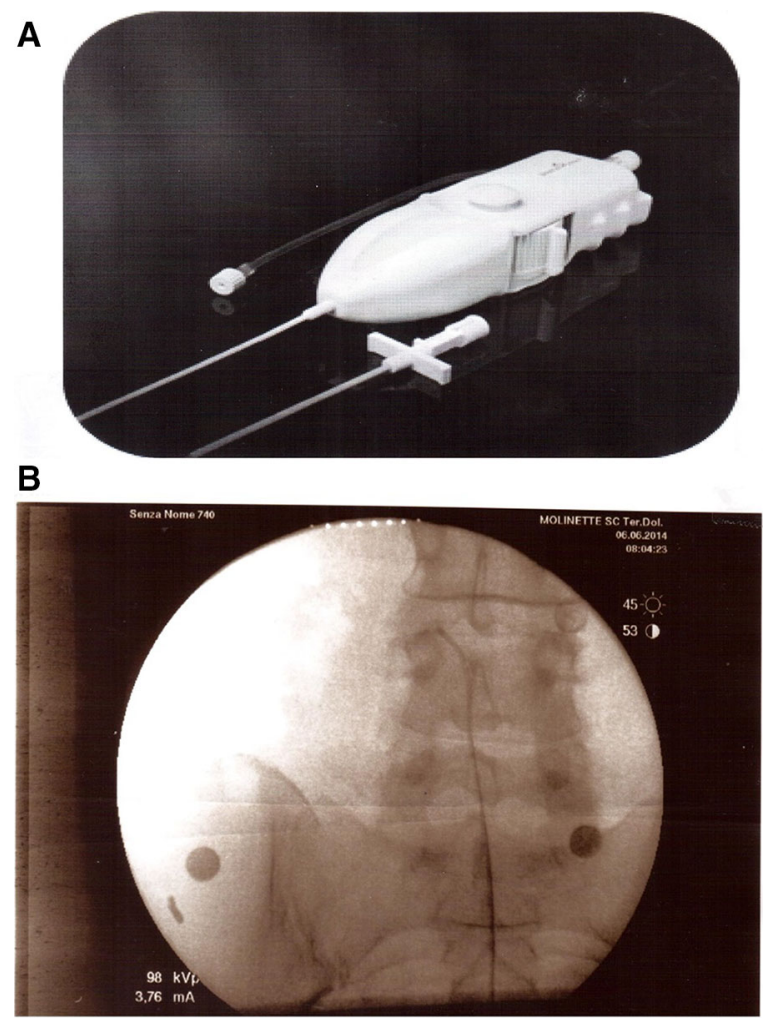

Fig. 1 a St. Reed kit: 15-gauge bone marrow needle $103 \mathrm{~mm}$ long, covered by a plastic cannula and an epidural catheter whose tip can be directed laterally. b Fluoroscopic image of epidural catheter: the tip of the catheter can be directed laterally 
The catheter and the peripheral venous catheter were removed and a small medication was applied. The patient laied for about $2 \mathrm{~h}$, then he was mobilized and dismissed the same day. The procedure was usually well tolerated and patients did not require sedative drugs. Intra or postoperative infusion of paracetamol was performed when needed.

\section{Measures and Statistical Analyses}

Patients were evaluated at a basal visit before the procedure, then by recurring control visits at $1,3,6$, and 12 months after the procedure. NRS scale was obtained at basal visit and in all follow-up visits. Pain assessment was performed at each follow-up visit and included: referred pain relief (PR-excellent: $>$ or $=75 \%$; good: between $50 \%$ and $75 \%$; poor: $<50 \%$ or unchanged), evaluation of analgesic consumption (AC-unchanged, 50\% reduction and $100 \%$ reduction), observation of functional recovery (FR-gain of function/no gain of function).

Primary outcome measures considered for the study were NRS value and referred pain relief (PR). Secondary outcomes were analgesic consumption (AC) and functional recovery (FR). Descriptive measures (frequency, mean, and standard deviation) were used to describe patients' characteristics and patients' improvement at different time-points. Scatter plots and $T$ test for paired samples were used to evaluate the modification of pain measures at different consecutive time-points. Analyses were conducted using IBM SPSS Statistics 23. Statistical significance was considered for $p$ values lower than 0.05 .

\section{RESULTS}

Patients characteristic are described in Table 1. Three patients were excluded from the study because the epidural procedure was early interrupted after the appearance of cardiac side effects (sinus bradycardia possibly induced by vagal stimulation). No complications were reported in other patients. Most of the patients had only one epidural injection of hydrocortisone. Three patients $(6 \%)$ received additional treatment with epidural sacral injection.

Long-term evaluation was done in 33 patients because some others dropped out at follow-up. $T$ test for paired samples showed a significant reduction of NRS score at any time point from basal score $(p<0.000)$. NRS reduction further improved after 2 months (T2 vs. T1; $p=0.02$ ) and reached a clinically relevant target (NRS score $<4$ ); the improvement was maintained at the following time-points (T3 and T4), as shown in Fig. 2.

Excellent pain relief ( $>75 \%$ ) was reported by $25 \%(12 / 48)$ of patients after only 1 month and by $48.5 \%(16 / 33)$ after 2 months (T2). Good pain relief (between 50 and $75 \%$ ) was reported by $39.6 \%(19 / 48)$ of patients after 1 month and by $36.4 \%(12 / 33)$ at the second month. Poor pain relief $(<50 \%)$ was reported only in $9.1 \%$ (3/33) of patients after 12 months (T4) (Table 2). Two patients relapsed after 2 months and one patient relapsed at the fourth month. Two patients reported poor pain relief and a vertebral surgery was necessary. Pain relief was stable at any time up to 12 months (Fig. 3).

We observed a significant reduction in mean analgesic consumption (Fig. 3). Moreover, about $76 \%$ of the patients were drug free at 12 months and $13 \%$ of patients reduced analgesic consumption to $50 \%$. Only three patients (10\%) reported unchanged analgesic consumption at T4 (Table 2). Functional recovery was observed in $96.9 \%(32 / 33)$ patients at 12 months (Fig. 3).

\section{DISCUSSION}

Epidural injections are one of the most commonly used treatments for managing chronic low back pain and all above radicular pain [3]. Epidural injections are administered by accessing the lumbar epidural space by multiple routes, such as caudal, interlaminar, and transforaminal. The interlaminar approach is considered to deliver the medication near the assumed site of pathology; the transforaminal approach is considered the more target-specific modality requiring the smallest volume to reach 


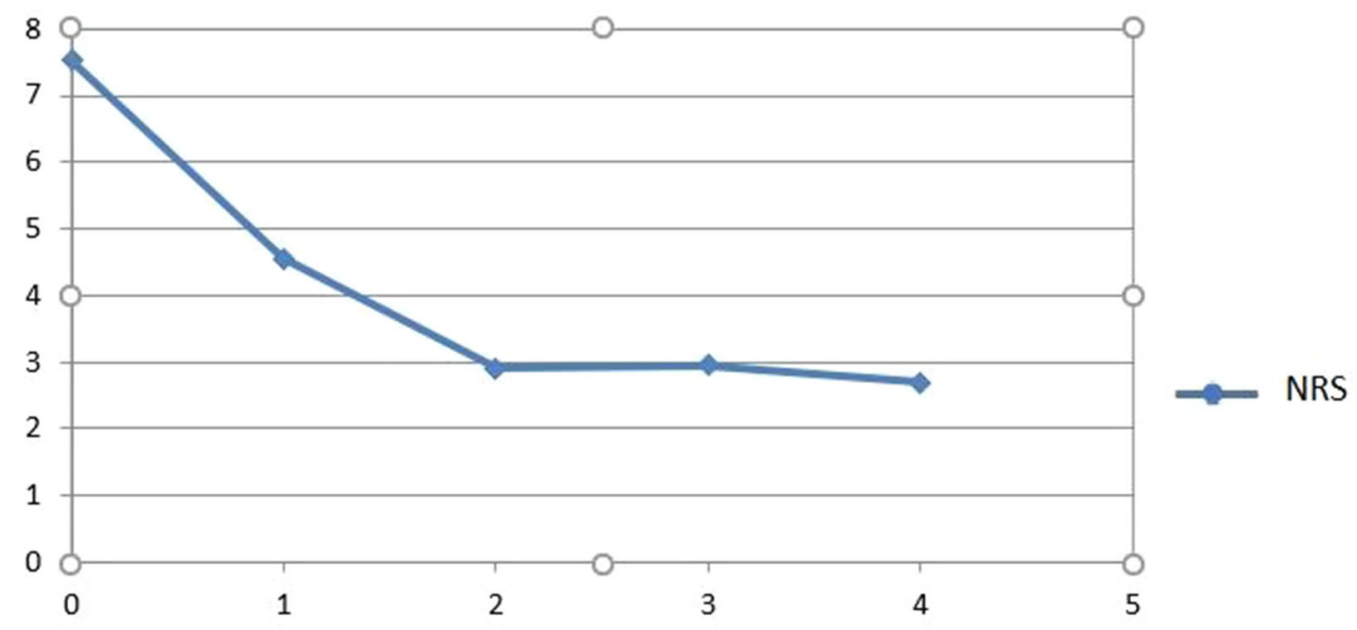

\begin{tabular}{|l|ccccc|}
\hline & T0 & T1 & T2 & T3 & T4 \\
\hline NRS (mean) & 7,53 & 4,56 & 2,90 & 2,95 & 2,70 \\
$\mathbf{p}$ & - & $.00 *$ & $.02 *$ & .89 & .67 \\
\hline
\end{tabular}

Fig. 2 Numerical rating scale (NRS) reduction at different time-points. $T$ test for paired samples; NRS reduction improved farther after the second treatment (T2 vs. T1), and the improvement was maintained at the following time-points (T2 vs. T3; T3 vs. T4). Statistical significance was considered for $p$ values lower than $0.05^{*}$

Table 2 Distribution of pain relief (PR) and analgesic reduction (AR) among patients at different time-points

\begin{tabular}{lllll}
\hline PR & \% $(\boldsymbol{N}$ of patients $)$ & & \\
\cline { 2 - 5 } & T1 & T2 & T3 & T4 \\
\hline Excellent $(>75 \%)$ & $25.0(12 / 48)$ & $48.5(16 / 33)$ & $60.6(20 / 33)$ & $60.6(20 / 33)$ \\
Good $(50-75 \%)$ & $39.6(19 / 48)$ & $36.4(12 / 33)$ & $27.2(9 / 33)$ & $27.2(9 / 33)$ \\
Poor $(<25 \%)$ & $35.4(17 / 48)$ & $9.1(3 / 33)$ & $12.1(4 / 33)$ & $9.1(3 / 33)$ \\
Relapse & - & $6.1(2 / 33)^{\mathrm{a}}$ & - & $1.8(1 / 33)^{\mathrm{a}}$ \\
AC reduction & $\mathrm{T} 1$ & $\mathrm{~T} 2$ & $\mathrm{~T} 3$ & $\mathrm{~T} 4$ \\
100\%-Drug free & $12.8(6 / 47)$ & $60.0(20 / 33)$ & $60.0(20 / 33)$ & $69.7(23 / 33)$ \\
$50 \%$ & $36.2(17 / 47)$ & $12.1(4 / 33)$ & $27.9(9 / 33)$ & $21.1(7 / 33)$ \\
Unchanged-relapse & $51.1(24 / 47)$ & $27.3(9 / 33)$ & $12.1(4 / 33)$ & $9.0(3 / 33)$ \\
\hline
\end{tabular}

a Three patients relapsed and received additional treatment with epidural sacral injection

the primary site of pathology. Caudal epidural injections are considered the safest and the easiest, with minimal risk of inadvertent dural puncture, even if they require relatively high volumes $[12,13]$. Recent literature has shown that even if it is less target-specific, the caudal 


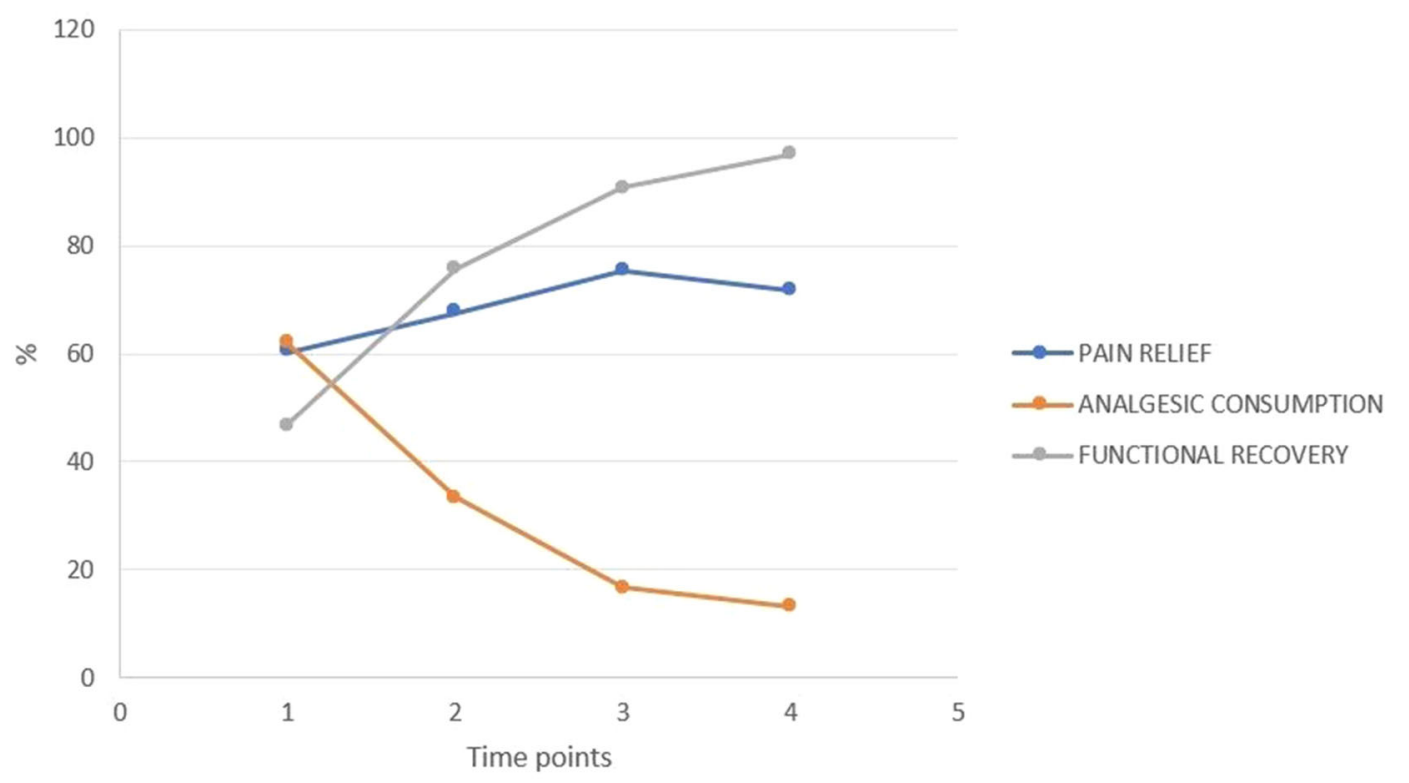

\begin{tabular}{|l|cccc|}
\hline & T1 & T2 & T3 & T4 \\
\hline PR (p) & 60,2 & $67,6(.11)$ & $75,4(.16)$ & $71,8(.53)$ \\
AC (p) & 62,1 & $33,3\left(.00^{*}\right)$ & $16,7(.26)$ & $13,0(.33)$ \\
FR & 46,7 & 75,7 & 90,9 & 96,9 \\
\hline
\end{tabular}

Fig. $3 T$ test for paired samples for pain relief (PR-\%) and mean analgesic consumption (AC-\%); (T1 vs. T2; T2 vs. T3; T3 vs. T4); statistical significance was

approach may provide equal effectiveness of either interlaminar and transforaminal injections $[3,12,13]$.

The evidence-based guidelines for interventional techniques in the diagnosis and treatment of chronic spinal pain provide recommendations for managing disc herniation or radiculitis beyond axial or discogenic pain without disc herniation, radiculitis, or facet joint pain. The recommendation is one of the three approaches that may be used [4].

Notwithstanding, even if different systematic reviews report that traditional caudal epidural approach is an effective management of chronic low back pain caused by disc herniation with radiculitis $[3,13,14]$ some observations should be made. In fact, many different work reports where up to $70-80 \%$ of patients express pain relief do not provide useful and compelling information on effectiveness, and considered for $p$ values lower than $0.05^{*}$. Increased percentage of patients with functional recovery (FR) at following timepoints

only a few studies provide information on secondary outcomes such as disability or function and use of other healthcare [3]. Some claim success rates based on improvements that are less than the minimal clinically important change for lumbar radicular pain [3, 4]. Moreover, many studies report up to five epidural injections are necessary to gain an acceptable pain relief $[3,15]$.

Some authors consider the transforaminal approach for epidural steroid injection the most effective technique for managing acute severe radicular pain because of its high target specificity [7]. Unfortunately, this technique can be associated with permanent lower extremity paralysis [5-7]. This severe complication has been postulated to be related to embolization, direct injury, compression, or transection of either the artery of Adamkiewicz or the radicular artery $[7,16,17]$. Moreover, it has been 
demonstrated that there is no significant relationship between variables such as needle size, local anesthetic injected, contrast or volume injected, and it can also occur in tomographyguided procedures or under fluoroscopy [7]. Therefore, even if paralysis is a rare major complication, it is unpredictable, and there is no safe procedure that may for sure prevent it.

Epidural selective injection with caudal approach through an adjustable catheter is a procedure easy to perform and free from the possible major complications of the transforaminal injection of steroids, but it can be equally target-specific. In fact, the adjustable catheter may be directed in the epidural space up to the selected level and side. No contrast medium is needed because the procedure is safer than adhesiolysis and there is no risk of intravascular intake. Therefore, there is no risk of anaphylactic reactions. In our sample of patients, the procedure was well tolerated and no side effects were observed. The proximity of the adjustable catheter to the root evokes pain only for a few seconds until the steroid injection. For this reason, there is no need for local anesthetics injection and motor paresis can be avoided. Hence, the procedure can be easily performed in outpatients.

We observed a significant reduction of NRS score that was maintained at any time point. After 1 month, the majority of patients reported more than $50 \%$ of pain relief, which is the minimum amount of change in pain score to be clinically meaningful [4] and the effect improved further after 3 months. At 12 months, up to $88 \%$ of patients reported good or excellent pain relief. Only a few patients perceived poor pain relief and only three patients relapsed. Moreover, we observed a progressive reduction of analgesic consumption with more than half of the patients drug free at 12 months.

Previous works exploring traditional transforaminal procedure displayed similar results on pain relief, even if secondary outcomes (such as analgesic consumption and functional recovery) are often lacking [18]. A recent review from MacVicar et al. [18] reported that about $60 \%$ of patients with radicular pain treated with transforaminal approach seemed to achieve at least $50 \%$ relief from pain between 1 and 2 months, but only a few maintained this outcome for 12 months. In fact, beyond 1 month, the proportion of patients with continued relief diminishes, and only $25-40 \%$ of patients reported relief that lasted 12 months [19-22]. On the contrary, in our study, pain relief was kept up to 12 months in over $90 \%$ of the patients.

Moreover, it has been seen that $94 \%$ of the patients treated with transforaminal approach achieve a successful outcome after only one treatment; only $4 \%$ of patients require a second injection and the use of three or four injections is a rare event [18]. In the same way, we reported only three patients (6\%) who required additional treatment with epidural sacral injection.

The literature is divided as to which corticosteroid preparation should be used, the optimal dose, or the volume injected. Different studies report successful outcomes from epidural injection, using different agents at different doses, ranging between 40 and $80 \mathrm{mg}$ of methylprednisolone or the equivalent dose of triamcinolone, betamethasone, and dexamethasone [18]. There is no report of injection of low doses of hydrocortisone, as we reported in our work. That means that our procedure gave excellent results on pain relief with a hydrocortisone dose sensibly lower than equivalent doses of corticosteroid preparation usually reported in transforaminal procedure. This is possible because the caudal approach allows the injection directly in the epidural space and the adjustable catheter may be directed selectively on the injured side. The subsequent injection of saline solution makes the procedure more efficient because it facilitates drug distribution and absorption in the epidural space.

The possibility of using low doses of corticosteroid makes our procedure even safer because it can be easily performed in patients with metabolic or cardiac comorbidities, too. In fact, our patient sample did not report any systemic side effects of steroid injection, which may be displayed in traditional epidural approaches (such as iatrogenic diabetes, osteoporosis, glaucoma, flushing, blood pressure alteration, hypothalamic-pituitary-adrenal axis disorders) [23, 24]. 
Hence, we can say that epidural steroid injection by caudal approach with adjustable catheter is a novel technique, superior to other epidural injections techniques for managing acute severe radicular pain. In fact, it achieves excellent effects on pain relief similar but more lasting if compared to those reported in patients treated with transforaminal approaches. This effect may be explained by the fact that the transforaminal approach is highly target-specific, but it is an external approach, and it acts outside of the root canal. Conversely, the caudal approach with adjustable catheter gathers the advantage of the traditional caudal approach to act directly inside the epidural space and the peculiarity of transforaminal approach to be extremely target-specific. Its high specificity allows injecting very small doses of corticosteroids; therefore systemic side effects may be avoided and patients with metabolic, cardiac, or visual disorders may be easily treated. In addition, the adjustable catheter makes the procedure free from the risk of major complications.

However, the time necessary to perform the procedure can vary (from 10 to $60 \mathrm{~min}$ ); it also depends on patient compliance because it needs care and accuracy in managing the $15-\mathrm{G}$ needle. Maybe it would be useful to create a kit with smaller needles in order to make the procedure easier and faster in patients with poor compliance.

However, compared to the tomography-guided procedures, it is less time-consuming, less expensive, and entails negligible X-ray exposure.

The major limitation of this study is the retrospective analysis of clinical data. For this reason, many patients have been lost during follow-up, and this made the number of patients in our sample even smaller. Our study measured the effectiveness of the procedure, but the absence of a placebo-controlled group made it impossible to measure absolute effect size.

\section{CONCLUSIONS}

The caudal approach with adjustable catheter is a novel, safe, effective, and target-specific technique for epidural steroid injection for managing acute severe radicular pain. It is superior to other epidural injection techniques because it achieves a similar but longer-lasting effect on pain relief.

Moreover it is extremely target-specific, and thus allows the use of small doses of corticosteroids, avoiding systemic side effects. It can be easily performed in patients with metabolic, cardiac, or visual disorders, too. In addition, the adjustable catheter makes the procedure free from the risk of major complications such as permanent lower-extremity paralysis. Larger studies and clinical trials should be performed to confirm our preliminary data.

\section{ACKNOWLEDGEMENTS}

Funding. No funding or sponsorship was received for this study. Sponsorship for article publication fees were funded by TSS Medical SRL. All authors had full access to all of the data in this study and take complete responsibility for the integrity of the data and the accuracy of the data analysis.

Editorial Assistance. Editorial assistance in the preparation of this article was provided by Dr. Vincenzo Bonicalzi and Cecilia Bonicalzi. This assistance was not funded.

Authorship. All named authors meet the International Committee of Medical Journal Editors (ICMJE) criteria for authorship for this article, take responsibility for the integrity of the work as a whole, and have given their approval for this version to be published.

Disclosures. Maria Clemente, Carla Roero, Veronica Perlo, Elena Peila, and Anna De Luca have nothing to disclose.

Compliance with Ethics Guidelines. All procedures performed in the study were in accordance with the ethical standards of the institutional and/or national research committee and with the 1964 Helsinki Declaration and its later amendments or comparable ethical 
standards. Patients gave their informed consent for participation in the research study.

Data Availability. The datasets during and/ or analyzed during the current study are available from the corresponding author on reasonable request.

Open Access. This article is distributed under the terms of the Creative Commons Attribution-NonCommercial 4.0 International License (http://creativecommons.org/licenses/ by-nc/4.0/), which permits any noncommercial use, distribution, and reproduction in any medium, provided you give appropriate credit to the original author(s) and the source, provide a link to the Creative Commons license, and indicate if changes were made.

\section{REFERENCES}

1. Lee JH, Lee S. Clinical and radiological characteristics of lumbosacral lateral disc herniation in comparison with those of medial disc herniation. Medicine (Baltimore). 2016;95(7):e2733.

2. Ashbrook J, Rodgdakis N, Goodwin P, Yeowell G, Callaghan M. Management acute low back pain in the ED: a systematic review. Emerg Med J. 2017;34(12):A889.

3. Parr AT, Hameed H, Conn A, et al. Caudal epidural injections in the management of chronic low back pain: a systematic appraisal of the literature. Pain Phys. 2012;15(3):E159-98.

4. Manchikanti L, Falco FJ, Singh V, et al. An update of comprehensive evidence-based guidelines for interventional techniques in chronic spinal pain. Part I: introduction and general considerations. Pain Phys. 2013;16(2 Suppl):S1-48.

5. Bathia A, Flamer D, Shaah PS, Cohen SP. Transforaminal epidural steroid injections for treating lumbosacral radicular pain from herniated intervertebral discs: a systematic review and meta-analysis. Anesth Analg. 2016;122(3):857-70.

6. Liu J, Zhou $\mathrm{H}$, Lu L, et al. The effectiveness of transforaminal versus caudal routes for epidural steroid injections in managing lumbosacral radicular pain: a systematic review and meta-analysis. Medicine (Baltimore). 2016;95(18):e3373.
7. Atluri S, Glaser SE, Shah RV, Sudarshan G. Needle position analysis in cases of paralysis from transforaminal epidurals: consider alternative approaches to traditional technique. Pain Phys. 2013;16(4):321-34.

8. Manchicanti L, Cash KA, Mcmanus CD, Pampati V. Assessment of effectiveness of percutaneous adhesiolysis in managing low back pain secondary to lumbar central spinal canal stenosis. Int J Med Sci. 2013;10(1):50-9.

9. Lee JH, Lee SH. Clinical effectiveness of percutaneous adhesiolysis and predictive factors of treatment efficacy in patients with lumbosacral spinal stenosis. Pain Med. 2013;14:1497-504.

10. Jones KR, Vojir CP, Hutt E, Fink R. Determining mild, moderate, and severe pain equivalency across pain-intensity tools in nursing home residents. J Rehabil Res Dev. 2007;44(2):305-14.

11. Manchikanti L, Falco FJE, Sing V, et al. An update of comprehensive evidence-based guidelines for interventional techniques in chronic spinal pain. Part II: Guidance and recommendations. Pain Phys. 2013;16:S48-283.

12. Manchikanti L, Singh V, Derby R, et al. Reassessment of evidence synthesis of occupational medicine practice guidelines for interventional pain management. Pain Phys. 2008;11:393-482.

13. Conn A, Buenaventura RM, Datta S, Abdi S, Diwan $S$. Systematic review of caudal epidural injections in the management of chronic low back pain. Pain Phys. 2009;12(1):109-35.

14. Manchikanti L, Datta S, Gupta S, et al. A critical review of the American Pain Society clinical practice guidelines for interventional techniques: Part 2. Therapeutic interventions. Pain Phys. 2010;13:E215-64.

15. Manchikanti L, Cash KA, McManus CD, Pampati V, Smith HS. One year results of a randomized, double-blind, active controlled trial of fluoroscopic caudal epidural injections with or without steroids in managing chronic discogenic low back pain without disc herniation or radiculitis. Pain Phys. $2011 ; 14: 25-36$.

16. Murthy NS, Maus TP, Behrns CL. Intraforaminal location of the great anterior radiculomedullary artery (artery of Adamkiewicz): a retrospective review. Pain Med. 2010;11(12):1756-64.

17. Alleyne $\mathrm{CH}$ Jr, Cawley CM, Shengelaia GG, Barrow DL. Microsurgical anatomy of the artery of Adamkiewicz and its segmental artery. J Neurosurg. 1998;89(5):791-5. 
18. MacVicar J, King W, Landers MH, Bogduk N. The effectiveness of lumbar transforaminal injection of steroids: a comprehensive review with systematic analysis of the published data. Pain Med. 2013;14(1):14-28.

19. Cyteval C, Fescquet N, Thomas E, Decoux E, Blotman F, Taourel P. Predictive factors of efficacy of periradicular corticosteroid injections for lumbar radiculopathy. AJNR Am J Neuroradiol. 2006;27(5):978-82.

20. Kabatas S, Cansever T, Yilmaz C, et al. Transforaminal epidural steroid injection via a preganglionic approach for lumbar spinal stenosis and lumbar discogenic pain with radiculopathy. Neurol India. 2010;58(2):248-52.

21. Ghahreman A, Ferch R, Bogduk N. The efficacy of transforaminal injection of stero treatment of lumbar radicular pain.ids. Pain Med. 2010;11(8):1149-68.

22. Karppinen J, Malmivaara A, Kurunlahti M, et al. Periradicular infiltration for sciatica: a randomized controlled trial. Spine (Phila Pa 1976). 2001;26(9):1059-67.

23. Bellini M, Barbieri M. Systemic effects of epidural steroid injections. Anaesthesiol Intensive Ther. 2013;45(2):93-8.

24. Leary J, Swislocki A. Hypothalamic-pituitary-adrenal suppression and iatrogenic Cushing's syndrome as a complication of epidural steroid injections. Case Rep Endocrinol. 2013;2013:617042. 